\title{
Survey of Nurses' Career Status in Grade III-A Hospitals in Shaanxi Province
}

\author{
Jiao Wang ${ }^{1}$, Haiyun Gai ${ }^{2, *}$ \\ ${ }^{1}$ Shaanxi University of Chinese Medicine, Xianyang, China. \\ ${ }^{2}$ Xi'an Hospital of Traditional Chinese Medicine, Xi'an, Shaanxi, China.
}

\begin{abstract}
How to cite this paper: Jiao Wang, Haiyun Gai. (2021) Survey of Nurses' Career Status in Grade III-A Hospitals in Shaanxi Province. International Journal of Clinical and Experimental Medicine Research, 5(2), 202-207. DOI: 10.26855/ijcemr.2021.04.014
\end{abstract}

Received: March 10, 2021

Accepted: April 5, 2021

Published: April 20, 2021

*Corresponding author: Haiyun Gai, Xi'an Hospital of Traditional Chinese Medicine, Xi'an, Shaanxi, China.

Email: 1239849356@qq.com

\begin{abstract}
Objective: To understand the career status of nurses in Grade III-A hospitals in Shaanxi province, explore its influencing factors, and put forward corresponding countermeasures. Methods: A total of 2,322 nurses in 9 Grade III-A hospitals in Shaanxi province were investigated by general information questionnaire and career status evaluation scale. Results: The career status score of the respondents was $(86.39 \pm 15.37)$, and the average score of the items was (3.76 \pm 0.67$)$, which was in the middle level. There were statistically significant differences in career status among nurses of different ages, professional titles, working hours and further training $(P<0.05)$. Multiple comparisons between the LSD groups showed that the scores of the professional career status of nurses aged 30 to 40 , with the title of nursing supervisor and 10 to 15 years of working experience were the lowest among the groups. Multiple linear regression analysis showed that monthly income level is also the main factor affecting the score. Conclusion: The career status of nurses in Grade III-A hospitals 1 of Shaanxi province is at medium level. The managers should pay more attention to the nurses with medium qualifications, improve the income of nurses, carry out corresponding career management education and training, guide the nurses to correctly understand the nursing profession, and promote the development of nursing career.
\end{abstract}

\section{Keywords}

Nurses, Career Status, Influencing Factors

\section{Introduction}

Career refers to the overall process of continuous experience of work-related behaviors, activities, attitudes, desires and values in an individual's life [1]. The career status of nurses directly affects the quality of clinical nursing, the stability of the nursing talent team, and the development of the nursing profession [2]. The "National Nursing Career Development Plan (2016-2020)" [3] proposed: "The number of registered nurses per 1,000 population reached 3.14". By the end of 2017, the total number of registered nurses in China exceeded 3.8 million. There is still a big gap in the goals of the nurses. Generally speaking, the career development of nurses in China is not optimistic [4], clinical nurses have a high degree of job burnout [5], low occupational satisfaction [6], and a high turnover rate [7] seriously affect the development of clinical nursing. At present, the research on nurses' career in our country is still in its infancy [8]. In view of this, this study investigated the current status of their career status and analyzed the influencing factors of 2,322 nurses in Grade III-A hospitals in Shaanxi Province. The results are as follows. 


\section{Object and methods}

\subsection{Objects of Study}

The convenience sampling method was adopted to select 2,322 nurses from 9 Grade III-A hospitals in Shaanxi Province from December 2020 to January 2021 to conduct a questionnaire survey. The inclusion criteria: (1) On-the-job nurses who have obtained a nurse qualification certificate; (2) Informed consent and voluntary participation in this study. Exclusion criteria: (1) Those who are on vacation and studying abroad; (2) Those who are studying outside the hospital.

\subsection{Methods}

Two scales were used in the survey: (1) General information questionnaire, the main content includes gender, age, marital status, education, professional title, employment nature, working hours, monthly income level, participation in advanced training, etc. (2) Career status evaluation scale, compiled by Tong Tian [9] based on multi-person theory, with 4 dimensions, including 8 items for occupational identity, 5 items for occupational satisfaction, 9 items for organizational commitment, and willingness to stay 1 Items, a total of 23 items. The scale uses the Likert 5-level scoring method, which has good reliability and validity, and the score is directly proportional to the career status. The entire questionnaire process is conducted through the "Questionnaire Star" platform. A total of 2,330 questionnaires were issued, 7 invalid questionnaires were eliminated, and 2,322 valid questionnaires were recovered. The effective response rate of the questionnaires was $99.6 \%$.

\subsection{Statistical analysis}

SPSS 25.0 software was used for data statistics and analysis. Desptive analysis, t-test and analysis of variance were used for univariate analysis. Multiple LSD comparisons were performed for statistically significant factors, multiple linear regression analysis was used for multivariate analysis.

\section{Results}

\subsection{Research object's career status score}

The research object's career status score is $(86.39 \pm 15.37)$ points, and the score rate is $75.09 \%$, which is at a medium level. See Table 1 for details.

\subsection{Single factor analysis of nurses' career status score}

A univariate analysis of the scores of 9 nursing staff with different general characteristics showed that the differences in age, title, working hours, and refresher training status were statistically significant $(\mathrm{P}<0.05)$, and LSD was performed on the different groups Test, the specific results are shown in Table 2.

\subsection{Multi-factor analysis of the research object's career status score}

Take the career status score of the research object as the dependent variable, and take the different characteristics of the general data of the research object as the independent variable. Perform multiple linear regression analysis. The analysis results show that age, title, working hours, monthly income level, and further training are the research objects. The influencing factors of the difference in career status scores, the specific results are shown in Table 3.

Table 1. The score of nurses' career status $(\mathrm{N}=\mathbf{2 3 2 2})$

\begin{tabular}{cccc}
\hline Dimension & items & Score range & Total Score \\
\hline Professional identity & 8 & $8-40$ & $30.34 \pm 5.49$ \\
Professional satisfaction & 5 & $5-25$ & $17.67 \pm 4.48$ \\
Organizational commitment & 9 & $9-45$ & $33.80 \pm 7.02$ \\
Willingness to stay & 1 & $1-5$ & $5(5,5)$ \\
Career total score & 23 & $23-115$ & $86.39 \pm 15.37$ \\
\hline
\end{tabular}


Table 2. Single factor analysis of nurses"' career status score $(\mathrm{N}=\mathbf{2 3 2 2})$

\begin{tabular}{|c|c|c|c|c|c|c|}
\hline Item & Group & $\mathrm{N}$ & Career score & $\mathrm{t} / \mathrm{F}$ & $\mathrm{P}$ & LSD \\
\hline Age & $\begin{array}{l}\text { (1) }<25 \\
\text { (2) } 25 \sim \\
\text { (3) } 30 \sim \\
\text { (4) } 40 \sim \\
\text { (5) } 50 \sim\end{array}$ & $\begin{array}{c}284 \\
896 \\
963 \\
137 \\
42\end{array}$ & $\begin{array}{l}87.02 \pm 16.37 \\
86.87 \pm 15.16 \\
85.36 \pm 15.56 \\
88.88 \pm 13.89 \\
87.57 \pm 14.79\end{array}$ & 2.394 & 0.048 & (3) $<$ (2) (4) \\
\hline Job title & $\begin{array}{l}\text { (1)Junior title } \\
\text { (2)Intermediate title } \\
\text { (3) Senior title }\end{array}$ & $\begin{array}{c}604 \\
1,116 \\
602\end{array}$ & $\begin{array}{l}87.82 \pm 16.22 \\
83.96 \pm 15.02 \\
88.01 \pm 13.99\end{array}$ & 9.928 & $<0.001$ & (2) $<$ (1) (3) \\
\hline $\begin{array}{c}\text { Working } \\
\text { years }\end{array}$ & $\begin{array}{l}\text { (1) }<2 \\
\text { (2) } 2 \sim \\
\text { (3) } 5 \sim \\
\text { (4) } 10 \sim \\
\text { (5) } 15 \sim\end{array}$ & $\begin{array}{l}216 \\
523 \\
805 \\
501 \\
109 \\
168\end{array}$ & $\begin{array}{l}90.12 \pm 15.21 \\
86.69 \pm 15.30 \\
86.47 \pm 15.60 \\
83.80 \pm 15.58 \\
85.20 \pm 13.84 \\
88.79 \pm 13.70\end{array}$ & 6.459 & $<0.001$ & (4) (5) $<$ (3) (2) $<$ (6) (1) \\
\hline $\begin{array}{l}\text { Refresher } \\
\text { training }\end{array}$ & $\begin{array}{l}\text { (1)never } \\
\text { (2)Inside the hospital } \\
\text { (3) Other hospitals }\end{array}$ & $\begin{array}{c}822 \\
1,114 \\
386\end{array}$ & $\begin{array}{l}84.39 \pm 16.42 \\
88.21 \pm 14.59 \\
85.42 \pm 14.83\end{array}$ & 15.712 & $<0.001$ & (1) $<$ (3) $<$ (2) \\
\hline
\end{tabular}

Table 3. Results of multiple linear regression analysis of factors affecting nurses' career status

\begin{tabular}{ccccccc}
\hline & $\beta$ & SE & $\beta^{\prime}$ & $\mathrm{t}$ & $\mathrm{P}$ \\
\hline (constant) & 90.294 & 2.922 & - & 30.906 & $<0.001$ \\
age & 2.313 & 0.700 & 0.128 & 3.304 & 0.001 \\
Education & -1.669 & 0.724 & -0.051 & -2.306 & 0.021 \\
job title & -2.801 & 0.635 & -0.145 & -4.412 & -2.283 & 0.023 \\
Working years & -1.091 & 0.478 & -0.091 & 0.001 & 0.005 \\
Monthly income level & 1.200 & 0.429 & 0.066 & 2.798 & $<.832$ & $<0.001$ \\
Refresher training situation & 1.849 & 0.483 & 0.084 & & & \\
\hline
\end{tabular}

Notes: $\mathrm{R}^{2}=0.026, \mathrm{~F}=7.652, \mathrm{P}<0.001$.

\section{Discussion}

\subsection{The status quo of nursing staff's career status in 9 tertiary hospitals in Shaanxi Province}

It can be seen from Table 1 that the career status of 2,322 nurses in 9 Grade III-A hospitals in Shaanxi Province was $(86.39 \pm 15.37)$ points, and the score was $75.09 \%$, which was at a medium level, but higher than the survey results of the following nurses' career status. Including: Shang Linping [10-11] surveyed in 2009, the score of the third-class hospital nurses in Taiyuan City (77.60 \pm 12.72$)$ points, Liu Hong [12] surveyed in 2013, the score of Shandong Province ICU specialist nurses (79.19 \pm 11.63$)$ points, Liu Zhuqing [13] surveyed in 2016, the ICU nurses of a tertiary hospital with a score of $(80.03 \pm 13.74)$ points, Fang Fang [14] surveyed in Henan Province's third-class hospital operating room specialist nurses score $(82.17 \pm 9.97)$ points, from the above research. The survey time and results of the survey show that with the development of time, the progress of society, the continuous reform of medical institutions, the continuous improvement of nursing management policies and systems, the nurses' career status scores are getting higher and higher, indicating that nursing care in recent years Career development is getting better and better. The scores of the four dimensions of career status are all higher than the research results of Liu Hong [12], Fang Fang [14], and Hua Guizhen [15]. Among them, the score of willingness to stay is the highest, 
which is similar to Shang Linping [10] and Wang Li [16], Zhu Xuemei [17] and others' research results are similar, but there is a certain gap with the current high nurse turnover rate in our country [7]. This may be due to the fact that nurses have become accustomed to their work and have feelings about their work units and are willing to Devotion to the current job is related; it may also be related to the hypothetical tone used in the content of the entry "Assuming that your unit will do its best to meet your needs and arrange interesting jobs for you". The dimension with the lowest scoring rate is the occupational satisfaction dimension, which is similar to Wang Li's research [16] results on nurses with a master's degree in a tertiary hospital in Chongqing and Zhu Xuemei [17] on nurses in a general hospital in Tai'an, which reflects the three-level in Shaanxi Province. Hospital nurses are generally dissatisfied with their job income and professional reputation, prompting managers to appropriately increase nurses' income, calling on the society to correctly understand the nurse industry, and increasing the reputation of the nursing industry in society.

\subsection{Analysis on the influencing factors of research objects' career status}

This study shows that there is no significant difference in the career status scores of nurses of different genders, educational backgrounds, and the nature of employment. The nature of employment and educational background are consistent with the results of Shang Linping [10], which is different from the results of Cui Lijun [18]. In recent years, medical institutions have continuously reformed the personnel system, and the gap between nurses of different employment nature has gradually narrowed, and there is not much difference in the work content of nurses with different educational backgrounds in clinical practice. Table 3 shows that the difference in nurses' career status scores of different ages, titles, working years, and refresher training status is statistically significant $(\mathrm{P}<0.05)$. Multiple comparisons between LSD groups show that: in terms of age, the 30-40 age group has the lowest career status score, which is inconsistent with the research results of Liu Zhuqing [13]. The research shows that the older nurses have better career status; in terms of job titles, the career status of nurses with the title of chief nurse has the lowest score, which is different from the results of many studies [10, 16-19]. In terms of working years, nurses who have worked for 10 to 15 years have the lowest scores compared with other groups. Combining the above research results on age, job title and working hours, it is obvious that the group of nurses who are 30-40 years old, whose job title is chief nurse, and who have worked for 10-15 years, roughly overlap. Studies have shown that the degree of job burnout of nurses at this stage is relatively serious [20]. They mostly take on teaching and management work in the department, and face greater pressure and limited promotion opportunities. At the same time, they also bear children, wives, and children in the family. Mother's multiple roles. When the income from work cannot meet her needs in life and her personal value is not fully realized, her career development will be delayed or even stagnant, so her career status score is lower than other groups. Nurse, in terms of refresher training, nurses who have never participated in refresher training scored the lowest, and nurses who participated in hospital refresher training scored the highest. This may be related to participating in hospital refresher training to improve the comprehensive ability of nurses and benefit their career development in the hospital; the scores of nurses who have participated in out-of-hospital advanced training are lower than those of in-hospital-trained nurses. They may have broadened their horizons and felt the advantages of the advanced hospital in many aspects when they studied in the out-of-hospital advanced training. Related. The results of multiple linear regression analysis show that the monthly income level is also an important factor affecting the nurses' career status scores. This is similar to the research results of Wang $\mathrm{Li}$ [16] and Zhu Xuemei [17]. "I am satisfied with my work income" is consistent with that economic income is the basic guarantee for career development. Ma Xinfeng [21] and others have shown that the income of nursing staff is closely related to job burnout, and low-income nurses are prone to job burnout, which affects their careers. Career status reminds nursing managers that appropriate incentive mechanisms should be established to increase the remuneration of nurses to prevent job burnout.

In summary, the career status scores of 2,322 nursing staff in 9 Grade III-A hospitals in Shaanxi Province are at a medium level. Age, title, working hours, monthly income level, and refresher training are the factors that affect the career status scores of nursing staff. Among them, Nurses who are between 30 and 40 years old, have the title of supervisory nurse, and have worked for 10 to 15 years, have low career status scores. Hospital administrators should pay attention to the care and support of nurses at this stage, strengthen humanistic care, prevent and mitigate them. The psychology of job burnout. At the same time, the role of monthly income and advanced training on career development cannot be ignored. Studies [22-23] have shown that active and effective career planning and management of nursing staff can promote the development of modern medicine and stabilize clinical practice. The nursing team can improve the job satisfaction of nursing staff and reduce their willingness to leave. Therefore, in 
addition to improving the welfare and treatment of nurses, nursing staff should also be trained in corresponding career aspects in order to improve nursing staff's professional satisfaction and willingness to stay, stabilize the nursing team, and promote the healthy development of nursing career.

\section{Declaration}

All authors of this article declare they have no conflicts of interest.

\section{Acknowledgements}

This study was approved by the Shaanxi Provincial Department of Science and Technology (2020KRM141) and the Innovation project for graduate students of university-level scientific research projects of Shaanxi University of Traditional Chinese Medicine in 2020 for funding the project.

\section{References}

[1] Du, Y. (2006). Career management [M]. Beijing: China Development Press, 2006: 123-124.

[2] Zhang, N. (2008). Research on nurse job burnout [D]. Anhui Medical University, 2008.

[3] Health and Family Planning Commission of the People's Republic of China. National Nursing Care Development Plan (2016-2020) [R]. 2016-11-18.

[4] Gao, X., Bi, Y. Y. (2013). Research progress on the professional development of domestic nursing staff [J]. Journal of Nursing Science, 2013, 28(08): 94-96.

[5] Liang, X., Feng, X. L. (2017). Research status of nursing staff burnout in my country [J]. Chinese Nursing Management, 2017, 17(11): 1513-1517.

[6] Zhang, L. F., You, L. M., Liu, K., et al. (2014). The association of Chinese hospital work environment with nurse burnout, job satisfaction, and in-tention to leave [J]. Nurs Outlook, 2014, 62(2): 128-137.

[7] Zhao, M. H., Ge, X. D., Zhang Meng. (2018). Analysis of the correlation between clinical nurses' sense of professional benefit and willingness to quit [J]. Journal of PLA Hospital Management, 2018, 25(07): 601-603+613.

[8] Yang, A. H., Jin, L. F., Liang Hui. (2018). Research progress of nurses' career planning and management at home and abroad [J]. Nursing Research, 2018, 32(10): 1514-1616.

[9] Tong, T. (2006). Career development and planning [M]. Beijing: Knowledge Press, 2006: 5-6.

[10] Shang, L. P., Xu, J. J. (2010). Investigation and analysis of 339 nursing staff's career status and its influencing factors in Taiyuan City [J]. Chinese Journal of Nursing, 2010, 45(8): 736-738.

[11] Shang, L. P., Song, L. P., Zhao Xiaoyan. (2012). Research on the relationship between nurses' psychological capital and self-career management [J]. Chinese Nursing Education, 2012, 9(12): 533-535.

[12] Liu, H., Jing, X. B., Fang Xiaojie, et al. (2016). Investigation on the career status of critically ill specialist nurses in Shandong Province [J]. Chinese Journal of Practical Nursing, 2016, 32(25): 1978-1981.

[13] Liu, Z. Q. (2018). Analysis of influencing factors of ICU nursing staff's career status [J]. Evidence-based Nursing, 2018, 4(6): 510-514.

[14] Fang, F., Fu, L. P., Wang, S., et al. (2018). Path analysis of the influence of subjective well-being and social support of specialist nurses in operating room on career status [J]. Chinese Journal of Modern Nursing, 2018, 24(18): 2168-2172.

[15] Hua, G. Z., Wang, X. L., Ma, X. (2013). Investigation on the career status and influencing factors of clinical nurses in a tertiary hospital [J]. Journal of Ningxia Medical University, 2013, 35(2): 184-187.

[16] Wang, L., Mou, S. Y., and Gan, X. N. (2018). Analysis on the career status and influencing factors of nurses with master degree in tertiary hospitals in Chongqing City and its influencing factors [J]. Chinese Nursing Management, 2018, 18(08): 1062-1067.

[17] Zhu, X. M., Ni, M., Yang, Z. X. (2018). The influence of nurses’ psychological capital on their career status in Tai’an General Hospital [J]. Chinese Continuing Medical Education, 2018, 10(30): 76-79.

[18] Cui, L. J., Li, S. S., Li, X. P. (2012). Investigation and analysis of young nurses' career status [J]. Chinese Journal of Modern Nursing, 2012, 18(15): 1737-1739.

[19] Wang, L., Mou, S. Y., Chen, L. Q., et al. (2017). Meta-analysis of the main influencing factors of nurses' career development. Chinese Journal of Practical Nursing, 2017, 33(35): 2796-2800.

[20] Zhou, J. Z., Li, H., Chen, P. J., et al. (2011). Correlation analysis between job burnout and job stressors of junior nurses [J]. Chinese Journal of Practical Nursing, 2011, 27(5): 71-72. 
[21] Ma, X. F., Hu, S. H., Zhao, Q. (2018). The effect of subjective and objective income on job burnout of nursing staff in military hospitals [J]. Occupation and Health, 2018, 34(21): 2951-2954.

[22] Pool, I. A., Poell, R. F., Berings, M. G. (2015). Strategies for continuing professional development among younger, middle-aged, and older nurses: a biographical approach [J]. Int J Nurs Stud., 2015, 52(5): 939-950.

[23] Rodts, M. F., Lamb, K. V. (2006). Transforming your professional self: encouraging lifelong personal and professional growth [J]. Orthop Nurse in Staff Development, 2006, 22(5): 246-253. 